\title{
Vitamin D status and associated occupational factors in Korean wage workers: data from the 5th Korea national health and nutrition examination survey (KNHANES 2010-2012)
}

Harin Jeong ${ }^{1 *}$, Sujin Hong ${ }^{1}$, Yunjeong Heo ${ }^{1}$, Hosun Chun ${ }^{1}$, Daeseong Kim', Jongtae Park ${ }^{1}$ and Mo-yeol Kang ${ }^{2}$

\begin{abstract}
Objectives: Vitamin D deficiency is increasing worldwide. However, few studies have attempted to examine the vitamin D status of wage workers and the correlation between vitamin D deficiency and working conditions. Hence, we aimed to evaluate the prevalence of vitamin D deficiency and the association between occupational conditions and vitamin D deficiency among Korean wage workers.

Methods: Wage workers aged 20-65 years from the 5th Korea National Health and Nutrition Examination Survey (KNHANES 2010-2012; $n=5409$ ) were included in our analysis. We measured the prevalence of vitamin D deficiency and identified the correlations with the working conditions of these subjects.

Results: The prevalence of vitamin D deficiency in male and female subjects was $69.5 \%$ and $83.1 \%$, respectively. Among the male subjects, a significant correlation between vitamin D deficiency and working conditions was observed among shift workers, office workers, and permanent workers. No significant correlation with any type of working conditions was observed among female subjects.

Conclusion: The prevalence of vitamin D deficiency among Korean wage workers was very high and was found to correlate significantly with working conditions, likely because of insufficient exposure to sunlight associated with certain types of work. Wage workers require more frequent outdoor activity and nutrition management to maintain sufficient vitamin D level.
\end{abstract}

Keywords: Vitamin D deficiency, Shift work, Office work, Indoor work, KNHANES

\section{Introduction}

Vitamin D (25-hydroxyvitamin D; $25(\mathrm{OH})$ D) deficiency is common in the general Korean population, and the increasing trend in vitamin $\mathrm{D}$ deficiency is a worldwide phenomenon [1-3]. Vitamin D deficiency is thought to be caused by lifestyle factors such as indoor confinement for a considerable period of the day, consumption of an imbalanced diet and low-quality (nutrient-poor) food, and widespread use of sunblock. Furthermore, in many big cities, air pollution and blockage of sunlight by high-rise buildings also contributes to vitamin D deficiency $[4,5]$.

\footnotetext{
* Correspondence: kirelog@paran.com

'Department of Occupational Medicine, Korea University Hosipital, 123, Jeokgeum-ro, Ansan, Gyeonggido, Republic of Korea

Full list of author information is available at the end of the article
}

Workers with vitamin D deficiency often present with common symptoms such as non-specific weakening of the muscles and myalgia, and these symptoms may be confused for fibromyalgia or chronic fatigue syndrome. In many cases, the musculoskeletal diseases of workers are attributed to the intensity of the work they perform or to their posture at work, whereas, unfortunately, vitamin D deficiency is seldom considered the potential cause of the symptoms [6-10].

Among the limited number of studies on the topic, most have focused on the prevalence of vitamin D deficiency by job, rather than on establishing a concrete correlation with working conditions [11]. Among the studies on the relationship of vitamin D deficiency and working conditions, limited studies examined miners who work underground 
for long hours [12]. The theoretical probability of the correlation between vitamin D deficiency and working conditions, such as shift work, has been noted. For example, the lack of vitamin D may play a role in the potential biological mechanisms of shift work as a "carcinogen" [13]. However, despite the theoretical probability of such correlations between working conditions and vitamin D deficiency, few attempts to examine their actual epidemiological correlation have been carried out $[14,15]$. Clinical attention to vitamin $\mathrm{D}$ deficiency has been mainly focused on growth in children, fractures in the elderly, and decreased bone density [16-18]. Perhaps because the causes and treatment of vitamin D deficiency are simple and obvious, researchers have not considered the need to manage the working conditions of healthy workers as well [19].

Vitamin D is widely recognized as important for bone health and maintenance. Moreover, vitamin D, which is known mainly for its role in calcium homeostasis and musculoskeletal conditions such as rickets and osteomalacia, is now also thought to be involved in various disease and pathologic processes such as cancer, cardiovascular disorders, and inflammation. Recently, vitamin $\mathrm{D}$ has been reconceptualized as a "hormone" rather than just a "nutrient" [20,21]. As increasing the vitamin D levels of workers may improve their musculoskeletal status and reduce the risk of chronic diseases, including some cancers, autoimmune diseases, infectious diseases, and type 2 diabetes mellitus, we investigated here the status of vitamin D deficiency and the association between serum vitamin D levels and working conditions in Korean wage workers to identify correctable occupational factors [22-24].

\section{Materials and methods Subjects}

The Korea National Health and Nutrition Examination Survey (KNHANES) is one of the most representative surveys of the entire national population of the Republic of Korea. It is administered by the Ministry of Health and Welfare as a means to evaluate the status of health and nutrition of Koreans nationwide. The fifth survey (2010-2012) continued the use of the rolling sampling survey method. This survey separated the ordinary residential areas from areas containing apartments to apply two different sets of sampling frameworks (examining the residential registration data for the ordinary residential areas and surveying market price trends in the apartment areas). Based on the complex samples, each of these areas was internally stratified. Twenty households were surveyed per area, and each household participated in interviews, medical examinations, and nutritional examinations. Interview personnel with relevant training visited the sample households in the area to conduct surveys using structured questionnaires to gather demographic, socio-economic, occupational, and health status information.

Out of the total of 25,534 subjects who participated in the fifth survey (2010-2012), 5,686 subjects who identified themselves as "wage workers" and were aged 20-65 years were selected for this study. Of these subjects, those who answered the questions related to their working conditions with "I do not know" or did not answer $(\mathrm{n}=56)$, those who did not undergo a vitamin D examination $(n=166)$, and those who did not answer the questions regarding their income, drinking habits, and smoking status $(n=55)$ were excluded, resulting in 5,409 subjects included in our analysis.

\section{Classification of data}

For this study, data regarding age, income, marital status, education, occupation, whether they worked in shifts, working hours, and total household income were gathered from each of the subjects. The subjects were grouped into 20-29, 30-39, 40-49, and 50-65-year age groups, and were further classified into married and unmarried groups, and into "elementary or lower education", "middle school", "high school" and "college or above" groups. The income level data were calculated by classifying the subjects into quartiles by total household income.

For the question "Do you currently smoke?" the subjects who answered "Yes" were classified as "current smokers" whereas the remaining subjects were grouped as "ex-smokers and non-smokers". With regard to drinking habits, the subjects were grouped according to whether they had consumed alcohol more than once a month in the past year. As for the nutrition- and exercise-related questions, the subjects were grouped according to whether they had consumed dietary supplements for $\geq 2$ weeks in the past year and whether they performed medium-intensity exercise, accompanied by significant fatigue or shortness of breath, in more than two sessions that lasted for at least 20 minutes each over the past week on $\geq 3$ days in a week.

Vitamin D deficiency is defined by most experts as serum vitamin D levels $<20 \mathrm{ng} / \mathrm{mL}$ (50 nmol/L), although there is no consensus on the optimal levels of serum vitamin D [25-28]. Accordingly, in this study, a cutoff value of $20 \mathrm{ng} / \mathrm{mL}$ was used to divide the samples into the "deficiency" group and the "normal" group.

As for the type of occupation, the occupation classification code was used to group the subjects who worked as managers, experts/specialists, or office workers as "office workers"; those who worked in the service or sales sectors as "service workers"; and workers in the fields of agriculture or fishery, and related industries, assembly of machinery, machine operation, and simple labor as "manufacturing workers". Those who responded that they worked between 6 a.m. and 6 p.m. were classified 
as "daytime workers"; and those who worked in the afternoon (2 p.m. to midnight), at night (from 9 p.m. to 8 a.m. the following day), in regular rotation of shifts between the day shifts and the night shifts, in 24-h shifts, in segmented shifts (working more than two shifts a day), and in irregular shifts, were all classified as "shift workers". The number of hours the subjects worked was counted based on their answers to the relevant open question in the questionnaire. Based on their responses, they were grouped into $<40$ hours per week group (which is the legal limitation of working hours in Korea), the $40 \sim 52$ hours per week group (which included the legal overtime limitation), and the $52 \sim 60$ hours per week group, $\geq 60$ hours per week group. The workers who had a permanent job were classified "permanent workers", and the others were classified as "temporary workers". Finally, the workers who worked on a parttime basis were classified as "part-time workers," and those who answered that they worked full-time were classified as "full-time workers."

\section{Statistical analysis}

The design of the samples in the national survey was based on the complex sample design method. To achieve results without biases, the samples must be analyzed using weights, stratified variables, and cluster variables, which are the primary extraction units. Thus, in this study, the statistical data extracted from the fifth national survey were analyzed similarly, using the integrated weights, stratified variables, and cluster variables.

The overall characteristics of the subjects were calculated using frequencies and percentages by gender. The average vitamin $\mathrm{D}$ level and the correlation between the prevalence of vitamin $\mathrm{D}$ deficiency and the relevant variables were calculated and verified using the $t$ test and the chi-square test. By using logistic regression models, we calculated the odds ratios of vitamin D deficiency associated with the variables on working conditions. The variables showed statistical correlations with vitamin $D$ deficiency in the univariate analyses, and factors already known to affect the vitamin D level (alcohol, smoking, $\mathrm{BMI}$, etc.) were used in the multivariate logistic regression analysis. For the statistical analysis, SPSS version 18.0 (Chicago, IL, USA) was used for statistical analysis, and the statistical significance was set at $\mathrm{p}<0.05$.

\section{Ethics statement}

We used reconstructed dataset from the 5th Korean National Health and Nutrition Examination Survey (KNHANES 2010-2012). All participants in this survey signed an informed consent form and the survey was approved by the institutional review board of Centers for Disease Control and Prevention in Korea (IRB No. 201002CON-21-C, 2011-02CON-06-C, 2012-01EXP-01-2C).

\section{Results}

The general characteristics of the subjects without the weight applied are shown in Table 1. Of the 5409 subjects in total, 2868 (53.0\%) were men, while 2541 (47.0\%) were women. The subjects who reported that they worked in shifts accounted for $18.5 \%(532 / 2868)$ of the male subjects and $17.2 \%(437 / 2541)$ of the female subjects. Those who described themselves as permanent workers were $84.8 \%$ of the male and $72.1 \%$ of the female subjects. A higher proportion of the female subjects than the male subjects were part-time workers, and more women worked in the service industry. Those who worked under the legal limit for working hours (52 hours) comprised $68.8 \%$ of the male and $84.7 \%$ of the female subjects (Table 1 ).

The results of a chi-square analysis using weight are shown in Tables 2 and 3. The vitamin D deficiency prevalence of men and women, respectively, were $69.5 \%$ and 83.1\%. Among the male subjects, the prevalence of vitamin D deficiency showed a significant difference depending on the use of food supplements, marital status, and education level. Furthermore, working conditions such as shift work, permanent work, and occupation contributed to significant differences as well $(\mathrm{p}<0.05)$ (Table 2).

Among the female subjects, BMI, food supplements, marital status, family income, and education level contributed to significant differences in the prevalence of vitamin D deficiency. The permanent workers (84.7\%) showed a higher prevalence of vitamin $\mathrm{D}$ deficiency compared to the temporary workers $(78.8 \%)$, while the prevalence of vitamin D deficiency among the office workers was $88.1 \%$, which was considerably higher than that of the manufacturing workers (79.0\%) (Table 3).

The result of a logistic regression model analysis for the male subjects showed that the risk of vitamin D deficiency significantly increased with shift work, permanent work, and office work. After some variables were adjusted, the odds ratio of the shift workers (vs. daytime workers) was 1.456 (CI 1.089-1.946), while the odds ratio of the permanent workers (vs. temporary workers) was 1.420 (CI 1.019 - 1.979). The univariate analysis by occupation showed that the office workers and the service workers both showed significant correlations with vitamin D level. However, after adjustment, only the office workers showed a significant odds ratio of 1.478 (CI $1.098-1.990)$. Of the female subjects, the correlation between their working conditions and vitamin D deficiency disappeared, which made it impossible to identify a clear occupational factor for vitamin D deficiency (Table 4).

\section{Discussion}

This study confirmed that the prevalence of vitamin D deficiency was very high among the wage workers of Korea. Furthermore, we showed here that occupational factors such as shift work and office work were related 
Table 1 General characteristics of the subjects

\begin{tabular}{|c|c|c|c|c|c|c|c|}
\hline & & \multicolumn{2}{|l|}{ Male } & \multicolumn{2}{|c|}{ Female } & \multicolumn{2}{|l|}{ Total } \\
\hline & & $\mathrm{N}^{*}$ & $\%$ & $\mathbf{N}$ & $\%$ & $\mathrm{~N}$ & $\%$ \\
\hline Total (N) & & 2868 & & 2541 & & 5409 & \\
\hline \multirow[t]{4}{*}{ Age (years) } & $20-29$ & 385 & $13.4 \%$ & 548 & $21.6 \%$ & 933 & $17.2 \%$ \\
\hline & $30-39$ & 903 & $31.5 \%$ & 630 & $24.8 \%$ & 1533 & $28.3 \%$ \\
\hline & $40-49$ & 793 & $27.6 \%$ & 630 & $24.8 \%$ & 1423 & $26.3 \%$ \\
\hline & $50-65$ & 787 & $27.4 \%$ & 733 & $28.8 \%$ & 1520 & $28.1 \%$ \\
\hline \multirow[t]{3}{*}{ Body mass index (BMI) $(n=5393)$} & $<23$ & 1040 & $36.4 \%$ & 1472 & $58.1 \%$ & 2512 & $46.6 \%$ \\
\hline & $23-25$ & 741 & $25.9 \%$ & 489 & $19.3 \%$ & 1230 & $22.8 \%$ \\
\hline & $\geq 25$ & 1077 & $37.7 \%$ & 574 & $22.6 \%$ & 1651 & $30.6 \%$ \\
\hline \multirow[t]{2}{*}{ Dietary supplementation $(n=4590)$} & Yes & 922 & $40.3 \%$ & 1182 & $51.4 \%$ & 2104 & $45.8 \%$ \\
\hline & No & 1368 & $59.7 \%$ & 1118 & $48.6 \%$ & 2486 & $54.2 \%$ \\
\hline \multirow[t]{2}{*}{ Physical activity } & Yes & 254 & $8.9 \%$ & 223 & $8.8 \%$ & 477 & $8.8 \%$ \\
\hline & No & 2614 & $91.1 \%$ & 2317 & $91.2 \%$ & 4931 & $91.2 \%$ \\
\hline \multirow[t]{2}{*}{ Drinking } & Yes & 2249 & $78.4 \%$ & 1252 & $49.3 \%$ & 3501 & $64.7 \%$ \\
\hline & No & 619 & $21.6 \%$ & 1289 & $50.7 \%$ & 1908 & $35.3 \%$ \\
\hline \multirow[t]{2}{*}{ Smoking status } & None/ex-smoker & 1553 & $54.1 \%$ & 2372 & $93.3 \%$ & 3925 & $72.6 \%$ \\
\hline & Current smoker & 1315 & $45.9 \%$ & 169 & $6.7 \%$ & 1484 & $27.4 \%$ \\
\hline \multirow[t]{2}{*}{ Marital status } & Married & 2324 & $81.0 \%$ & 1910 & $75.2 \%$ & 4234 & $78.3 \%$ \\
\hline & Unmarried & 544 & $19.0 \%$ & 631 & $24.8 \%$ & 1175 & $21.7 \%$ \\
\hline \multirow[t]{4}{*}{ Income (quartile) } & Low & 154 & $5.4 \%$ & 216 & $8.5 \%$ & 370 & $6.8 \%$ \\
\hline & Middle low & 739 & $25.8 \%$ & 645 & $25.4 \%$ & 1384 & $25.6 \%$ \\
\hline & Middle high & 1020 & $35.6 \%$ & 837 & $32.9 \%$ & 1857 & $34.3 \%$ \\
\hline & High & 955 & $33.3 \%$ & 843 & $33.2 \%$ & 1798 & $33.2 \%$ \\
\hline \multirow[t]{4}{*}{ Education } & Less than elementary school & 121 & $4.2 \%$ & 285 & $11.2 \%$ & 406 & $7.5 \%$ \\
\hline & Middle school & 183 & $6.4 \%$ & 258 & $10.2 \%$ & 441 & $8.2 \%$ \\
\hline & High school & 889 & $31.0 \%$ & 831 & $32.7 \%$ & 1720 & $31.8 \%$ \\
\hline & More than college & 1675 & $58.4 \%$ & 1167 & $45.9 \%$ & 2842 & $52.5 \%$ \\
\hline \multirow[t]{2}{*}{ Shift work } & Yes & 532 & $18.5 \%$ & 437 & $17.2 \%$ & 969 & $17.9 \%$ \\
\hline & No & 2336 & $81.5 \%$ & 2104 & $82.8 \%$ & 4440 & $82.1 \%$ \\
\hline \multirow[t]{2}{*}{ Stability of work } & Permanent worker & 2432 & $84.8 \%$ & 1831 & $72.1 \%$ & 4263 & $78.8 \%$ \\
\hline & Temporary worker & 436 & $15.2 \%$ & 710 & $27.9 \%$ & 1146 & $21.2 \%$ \\
\hline \multirow[t]{2}{*}{ Employment type } & Part-time worker & 167 & $5.8 \%$ & 638 & $25.1 \%$ & 805 & $14.9 \%$ \\
\hline & Full-time worker & 2701 & $94.2 \%$ & 1903 & $74.9 \%$ & 4604 & $85.1 \%$ \\
\hline \multirow[t]{3}{*}{ Occupation } & Office worker & 1427 & $49.8 \%$ & 1232 & $48.5 \%$ & 2659 & $49.2 \%$ \\
\hline & Service worker & 291 & $10.1 \%$ & 598 & $23.5 \%$ & 889 & $16.4 \%$ \\
\hline & Manufacturing worker & 1150 & $40.1 \%$ & 711 & $28.0 \%$ & 1861 & $34.4 \%$ \\
\hline \multirow[t]{4}{*}{ Working hours/week } & $<40$ & 413 & $14.4 \%$ & 963 & $37.9 \%$ & 1376 & $25.4 \%$ \\
\hline & $40-52$ & 1561 & $54.4 \%$ & 1188 & $46.8 \%$ & 2749 & $50.8 \%$ \\
\hline & $52-60$ & 332 & $11.6 \%$ & 188 & $7.4 \%$ & 520 & $9.6 \%$ \\
\hline & $\geq 60$ & 562 & $19.6 \%$ & 202 & $7.9 \%$ & 764 & $14.1 \%$ \\
\hline
\end{tabular}

*Unweighted count.

to an increased risk of vitamin D deficiency in the male subjects. Only a few previous studies have suggested correlations between vitamin D deficiency and working conditions. In 2011, a British cohort study revealed that women, but not men, working at night and longer hours might be vulnerable to deficits in vitamin $\mathrm{D}$ and to the 
Table 2 Vitamin D status according to variables (Male)

\begin{tabular}{|c|c|c|c|c|c|c|c|}
\hline & & \multirow{3}{*}{$\begin{array}{l}\text { Vitamin D }(\mathrm{ng} / \mathrm{ml}) \\
\text { Mean (S.E) }\end{array}$} & \multicolumn{4}{|c|}{ Vitamin D } & \multirow[t]{3}{*}{ p-value } \\
\hline & & & \multicolumn{2}{|c|}{$\geq 20 \mathrm{ng} / \mathrm{ml}$} & \multicolumn{2}{|c|}{$<20 \mathrm{ng} / \mathrm{ml}$} & \\
\hline & & & $\mathrm{N}^{\S}$ & $\%$ (S.E) & $\mathrm{N}$ & $\%(S . E)$ & \\
\hline Total & & $17.8(0.175)$ & 7434830 & $30.5(1.3)$ & 16921262 & $69.5(1.3)$ & \\
\hline \multirow[t]{4}{*}{ Age (years) } & $20-29$ & $16.7(0.314)$ & 1204327 & $22.8(2.6)$ & 4068221 & $77.2(2.6)$ & $<0.001$ \\
\hline & $30-39$ & $17.3(0.240)$ & 2139963 & $27.5(1.9)$ & 5636522 & $72.5(1.9)$ & \\
\hline & $40-49$ & $18.3(0.284)$ & 2230488 & $34.0(2.4)$ & 4330483 & $66.0(2.4)$ & \\
\hline & $50-65$ & $19.2(0.291)$ & 1860052 & $39.2(2.3)$ & 2886036 & $60.8(2.3)$ & \\
\hline \multirow[t]{3}{*}{ Body mass index (BMI) $(n=5393)$} & $<23$ & $17.7(0.248)$ & 2706131 & 29.7(1.8) & 6390223 & 70.3(1.8) & 0.711 \\
\hline & $23-25$ & $18.1(0.250)$ & 1959023 & $31.9(2.2)$ & 4177771 & $68.1(2.2)$ & \\
\hline & $\geq 25$ & $17.8(0.229)$ & 2769676 & $30.7(1.9)$ & 6263714 & 69.3(1.9) & \\
\hline \multirow[t]{2}{*}{ Dietary supplementation $(n=4590)$} & Yes & $18.4(0.226)$ & 3515136 & $35.1(2.0)$ & 8538062 & $64.9(2.0)$ & 0.015 \\
\hline & No & 17.6(0.226) & 2489982 & $29.2(1.7)$ & 4608936 & 70.8(1.7) & \\
\hline \multirow[t]{2}{*}{ Physical activity } & Yes & 18.8(0.463) & 942016 & $38.5(3.7)$ & 1501849 & 61.5(3.7) & 0.17 \\
\hline & No & $17.7(0.178)$ & 6492814 & 29.6(1.4) & 15419414 & 70.4(1.6) & \\
\hline \multirow[t]{2}{*}{ Drinking } & Yes & $17.8(0.177)$ & 5860951 & $30.6(1.4)$ & 13320375 & 69.4(1.4) & 0.958 \\
\hline & No & $17.7(0.348)$ & 1573879 & $30.4(2.6)$ & 3600887 & $69.6(2.6)$ & \\
\hline \multirow[t]{2}{*}{ Smoking status } & None/ex-smoker & $17.9(0.194)$ & 3787159 & $30.7(1.6)$ & 8534766 & 69.3(1.6) & 0.828 \\
\hline & Current smoker & $17.7(0.228)$ & 3647671 & $30.3(1.7)$ & 8386496 & 69.3(1.7) & \\
\hline \multirow[t]{2}{*}{ Marital status } & Married & 18.3(0.186) & 5891190 & $33.7(1.5)$ & 11592550 & $66.3(1.5)$ & $<0.001$ \\
\hline & Unmarried & $16.6(0.281)$ & 1543640 & $22.5(2.0)$ & 5328712 & $77.5(2.0)$ & \\
\hline \multirow[t]{4}{*}{ Income (quartile) } & Low & $18.1(0.541)$ & 549598 & $34.8(4.8)$ & 1027913 & $65.2(4.8)$ & 0.546 \\
\hline & Middle low & 18.0(0.299) & 2183872 & $31.9(2.4)$ & 4670497 & $68.1(2.4)$ & \\
\hline & Middle high & $17.6(0.232)$ & 2641618 & $29.9(1.8)$ & 6183484 & $70.1(1.8)$ & \\
\hline & High & $17.9(0.228)$ & 2059742 & $29.0(1.8)$ & 5039367 & 71.0(1.8) & \\
\hline \multirow[t]{4}{*}{ Education } & Less than elementary school & $19.1(0.682)$ & 359510 & $40.1(6.2)$ & 536938 & $59.9(6.2)$ & $<0.001$ \\
\hline & Middle school & 19.4(0.523) & 600130 & 43.6(4.3) & 777380 & $56.4(4.3)$ & \\
\hline & High school & 18.3(0.296) & 2782314 & $34.8(2.1)$ & 5208570 & $65.2(2.1)$ & \\
\hline & More than college & $17.3(0.189)$ & 3692876 & $26.2(1.5)$ & 10398374 & $73.8(1.5)$ & \\
\hline \multirow[t]{2}{*}{ Shift work } & Yes & $17.0(0.316)$ & 1119179 & $24.4(2.3)$ & 3458301 & $75.6(2.3)$ & 0.004 \\
\hline & No & 18.0(0.185) & 6315651 & $31.9(1.5)$ & 13462961 & $68.1(1.5)$ & \\
\hline \multirow[t]{2}{*}{ Stability of work } & Permanent worker & $17.6(0.171)$ & 5746351 & $28.9(1.3)$ & 14165812 & $71.1(1.3)$ & 0.003 \\
\hline & Temporary worker & 18.8(0.379) & 1688479 & $38.0(3.2)$ & 2755451 & $62.0(3.2)$ & \\
\hline \multirow[t]{2}{*}{ Employment type } & Part-time worker & $17.8(0.542)$ & 493566 & 28.4(4.3) & 1245957 & 71.6(4.3) & 0.593 \\
\hline & Full-time worker & $17.8(0.174)$ & 6941264 & $30.7(1.3)$ & 15675306 & $69.3(1.3)$ & \\
\hline \multirow[t]{3}{*}{ Occupation } & Office worker & $17.3(0.192)$ & 2918874 & $26.2(1.6)$ & 8205357 & $73.8(1.6)$ & $<0.001$ \\
\hline & Service worker & $17.1(0.375)$ & 768684 & 26.2(3.4) & 2164465 & 73.8(3.4) & \\
\hline & Manufacturing worker & $18.6(0.273)$ & 3747272 & $36.4(2.0)$ & 6551440 & $63.6(2.0)$ & \\
\hline \multirow[t]{4}{*}{ Working hours/week } & $<40$ & $17.9(0.365)$ & 1188552 & $31.4(2.7)$ & 2595496 & $68.6(2.7)$ & 0.874 \\
\hline & $40-52$ & $17.7(0.211)$ & 3820692 & $29.8(1.7)$ & 9001176 & $70.2(1.7)$ & \\
\hline & $52-60$ & 18.0(0.379) & 942670 & $32.3(3.4)$ & 1976736 & $67.7(3.4)$ & \\
\hline & $\geq 60$ & $17.9(0.290)$ & 1482916 & $30.7(2.5)$ & 3347854 & $69.3(2.5)$ & \\
\hline
\end{tabular}

S.E; standard error, § estimated population size. 
Table 3 Vitamin D status according to variables (Female)

\begin{tabular}{|c|c|c|c|c|c|c|c|}
\hline & & \multirow{3}{*}{$\begin{array}{l}\text { Vitamin D }(\mathrm{ng} / \mathrm{ml}) \\
\text { Mean (S.E) }\end{array}$} & \multicolumn{4}{|c|}{ Vitamin D } & \multirow[t]{3}{*}{ p-value } \\
\hline & & & \multicolumn{2}{|c|}{$\geq 20 \mathrm{ng} / \mathrm{ml}$} & \multicolumn{2}{|c|}{$<20 \mathrm{ng} / \mathrm{ml}$} & \\
\hline & & & $\mathrm{N}^{\S}$ & $\%$ (S.E) & $\mathrm{N}$ & $\%(S . E)$ & \\
\hline Total & & 15.6(0.163) & 2748248 & $16.9(1.1)$ & 13530639 & 83.1(1.1) & \\
\hline \multirow[t]{4}{*}{ Age (years) } & $20-29$ & $14.1(0.251)$ & 383860 & $8.4(1.6)$ & 4159095 & 91.6(1.6) & $<0.001$ \\
\hline & $30-39$ & $15.3(0.283)$ & 542108 & 14.9(1.9) & 3098926 & 85.1(1.9) & \\
\hline & $40-49$ & $15.6(0.268)$ & 724950 & $16.7(2.0)$ & 3613641 & $83.3(2.0)$ & \\
\hline & $50-65$ & $17.5(0.271)$ & 1097330 & $29.2(2.1)$ & 2658978 & $70.8(2.1)$ & \\
\hline \multirow[t]{3}{*}{ Body mass index (BMI) $(n=5393)$} & $<23$ & $15.3(0.195)$ & 1457436 & 15.3(1.2) & 8095076 & $84.7(1.2)$ & 0.033 \\
\hline & $23-25$ & $16.2(0.293)$ & 642163 & $21.2(2.2)$ & 2382941 & $78.8(2.2)$ & \\
\hline & $\geq 25$ & $15.5(0.249)$ & 631525 & 17.3(1.9) & 3026934 & $82.7(1.9)$ & \\
\hline \multirow[t]{2}{*}{ Dietary supplementation $(n=4590)$} & Yes & $16.4(0.224)$ & 1587530 & $22.1(1.6)$ & 5583799 & 77.9(1.6) & $<0.001$ \\
\hline & No & $14.7(0.194)$ & 874424 & $12.0(1.2)$ & 6426624 & $88.0(1.2)$ & \\
\hline \multirow[t]{2}{*}{ Physical activity } & Yes & $16.4(0.440)$ & 260879 & 19.0(3.2) & 1110135 & 81.0(3.2) & 0.455 \\
\hline & No & $15.5(0.164)$ & 2487369 & 16.7(1.1) & 12415666 & 83.3(1.1) & \\
\hline \multirow[t]{2}{*}{ Drinking } & Yes & $15.7(0.212)$ & 1380747 & 16.0(1.4) & 7252876 & $84.0(1.4)$ & 0.298 \\
\hline & No & 15.4(0.199) & 1367501 & $17.9(1.4)$ & 6277763 & $82.1(1.4)$ & \\
\hline \multirow[t]{2}{*}{ Smoking status } & None/ex-smoker & $15.5(0.161)$ & 2495022 & 16.7(1.0) & 12432922 & 83.3(1.0) & 0.564 \\
\hline & Current smoker & $15.7(0.498)$ & 253226 & 18.7(3.8) & 1097717 & 81.3(3.8) & \\
\hline \multirow[t]{2}{*}{ Marital status } & Married & $16.2(0.187)$ & 2321272 & $20.4(1.3)$ & 9058540 & 79.6(1.3) & $<0.001$ \\
\hline & Unmarried & $14.1(0.234)$ & 426976 & $8.7(1.3)$ & 4472099 & 91.3(1.3) & \\
\hline \multirow[t]{4}{*}{ Income (quartile) } & Low & $16.9(0.502)$ & 368499 & 27.6(3.7) & 968252 & 72.4(3.7) & $<0.001$ \\
\hline & Middle low & $15.9(0.287)$ & 932271 & 21.3(2.1) & 3434927 & $78.7(2.1)$ & \\
\hline & Middle high & $15.3(0.234)$ & 758403 & $13.7(1.5)$ & 4762438 & $86.3(1.5)$ & \\
\hline & High & $15.3(0.234)$ & 689075 & $13.6(1.5)$ & 4365022 & $86.4(1.5)$ & \\
\hline \multirow[t]{4}{*}{ Education } & Less than elementary school & $17.5(0.366)$ & 467909 & $30.7(3.1)$ & 1056651 & 69.3(3.1) & $<0.001$ \\
\hline & Middle school & $17.1(0.436)$ & 370744 & 24.0(2.9) & 1174167 & $76.0(2.9)$ & \\
\hline & High school & $15.3(0.240)$ & 978233 & $17.2(1.8)$ & 4698721 & $82.8(1.8)$ & \\
\hline & More than college & $15.0(0.198)$ & 931362 & $12.4(1.2)$ & 6601101 & $87.6(1.2)$ & \\
\hline \multirow[t]{2}{*}{ Shift work } & Yes & $14.8(0.314)$ & 443644 & $14.4(2.1)$ & 2637092 & $85.6(2.1)$ & 0.183 \\
\hline & No & $15.7(0.172)$ & 2304604 & $17.5(1.2)$ & 10893547 & $82.5(1.2)$ & \\
\hline \multirow[t]{2}{*}{ Stability of work } & Permanent worker & $15.3(0.184)$ & 1810551 & $15.3(1.2)$ & 10038350 & $84.7(1.2)$ & 0.01 \\
\hline & Temporary worker & $16.3(0.254)$ & 937697 & $21.2(2.1)$ & 3492290 & $78.8(2.1)$ & \\
\hline \multirow[t]{2}{*}{ Employment type } & Part-time worker & $16.0(0.280)$ & 830474 & $21.0(2.1)$ & 3127546 & $79.0(2.1)$ & 0.014 \\
\hline & Full-time worker & $15.4(0.177)$ & 1917774 & $15.6(1.2)$ & 10403093 & $84.4(1.2)$ & \\
\hline \multirow[t]{3}{*}{ Occupation } & Office worker & $15.0(0.203)$ & 945750 & $11.9(1.2)$ & 6993107 & $88.1(1.2)$ & $<0.001$ \\
\hline & Service worker & $15.9(0.304)$ & 784657 & $19.9(2.1)$ & 3154425 & $80.1(2.1)$ & \\
\hline & Manufacturing worker & $16.3(0.262)$ & 1017841 & $23.1(2.0)$ & 3383107 & $76.9(2.0)$ & \\
\hline \multirow[t]{4}{*}{ Working hours/week } & $<40$ & $16.1(0.217)$ & 1168254 & 19.9(1.7) & 4701664 & $80.1(1.7)$ & 0.029 \\
\hline & $40-52$ & $15.2(0.201)$ & 1077340 & 14.1(1.2) & 6549011 & $85.9(1.2)$ & \\
\hline & $52-60$ & $15.3(0.472)$ & 243282 & $17.8(3.5)$ & 1126308 & $82.2(3.5)$ & \\
\hline & $\geq 60$ & $15.2(0.435)$ & 259372 & $18.4(3.2)$ & 1153657 & $81.6(3.2)$ & \\
\hline
\end{tabular}

S.E; standard error, § estimated population size. 
Table 4 Logistic regression analyses of working conditions

\begin{tabular}{|c|c|c|c|c|c|}
\hline & & \multicolumn{2}{|l|}{ Male } & \multicolumn{2}{|l|}{ Female } \\
\hline & & Crude OR(95\% Cl) & Adjusted OR(95\% Cl)* & Crude OR(95\% Cl) & Adjusted OR(95\% Cl)* \\
\hline \multirow[t]{2}{*}{ Shift work } & Yes & $1.449(1.119-1.876)$ & $1.456(1.089-1.946)$ & $1.258(0.893-1.773)$ & $1.357(0.920-2.004)$ \\
\hline & No & Reference & Reference & Reference & Reference \\
\hline \multirow[t]{2}{*}{ Stability of work } & Permanent worker & $1.511(1.158-1.971)$ & $1.420(1.019-1.979)$ & $1.489(1.105-2.006)$ & $0.977(0.685-1.392)$ \\
\hline & Temporary worker & Reference & Reference & Reference & Reference \\
\hline \multirow[t]{2}{*}{ Employment type } & Part-time worker & $1.118(0.740-1.688)$ & $1.086(0.641-1.841)$ & $0.694(0.521-0.924)$ & $0.844(0.578-1.234)$ \\
\hline & Full-time worker & Reference & Reference & Reference & Reference \\
\hline \multirow[t]{3}{*}{ Occupation } & Office worker & $1.608(1.302-1.985)$ & $1.478(1.098-1.990)$ & $2.225(1.657-2.986)$ & $1.141(0.752-1.730)$ \\
\hline & Service worker & $1.611(1.105-2.347)$ & $1.202(0.788-1.834)$ & $1.209(0.882-1.659)$ & $1.052(0.740-1.497)$ \\
\hline & Manufacturing worker & Reference & Reference & Reference & Reference \\
\hline \multirow[t]{4}{*}{ Working hours/week } & $<40$ & $0.967(0.703-1.331)$ & $1.077(0.720-1.612)$ & $0.905(0.579-1.415)$ & $0.965(0.564-1.652)$ \\
\hline & $40-52$ & $1.044(0.812-1.341)$ & $0.927(0.701-1.225)$ & $1.367(0.874-2.137)$ & $1.025(0.601-1.746)$ \\
\hline & $52-60$ & $0.929(0.638-1.352)$ & $0.916(0.616-1.364)$ & $1.041(0.571-1.897)$ & $0.684(0.348-1.344)$ \\
\hline & $\geq 60$ & Reference & Reference & Reference & Reference \\
\hline
\end{tabular}

*OR; odds ratio, $\mathrm{Cl}$; confidence intervals.

Adjusted for age, BMI, socioeconomic status (income, marriage, education level).

Health behavior (smoking status, alcohol use, dietary supplement use, physical activity).

associated health hazards. According to the authors, that was the first study exploring the relationship between different occupations and vitamin D deficiency [14].

It seems that the differences in the risk for vitamin $D$ deficiency in subjects working in shifts and according to the type of work (e.g. office work) are directly related to exposure to sunlight. Vitamin D can either be absorbed from food or naturally synthesized in the body upon skin exposure to sunlight. It is stored in the human body as a precursor vitamin D molecule, which is converted to the active and free form when it is exposed to ultraviolet B (UV-B) radiation (wavelength, 280-320 nm). Active vitamin D helps the body to absorb calcium and controls the density of calcium in our body [29].

Approximately $90 \%$ of the vitamin D in our body is generated upon exposure to sunlight, and the remaining $10 \%$ can be absorbed from ingested food. Among UV bands, only UV-B can trigger the synthesis of vitamin D. UV-B cannot penetrate glass; therefore, exposure to sunlight indoors through a window does not produce vitamin $\mathrm{D}$. As a general rule, the more oblique the incidence angle at which sunlight passes, the more UV-B light is absorbed; therefore, only sunlight produced in the middle of the day (between about 10:00 and 15:00) is effective for synthesis of vitamin $D$, and if people are not exposed to adequate sunlight during this time, they may be at risk of developing vitamin D deficiency [30,31].

Interestingly, among the male subjects, the permanent workers showed a higher prevalence of vitamin D deficiency than the temporary workers. The male permanent workers worked an average of 49.2 hours/a week, while the temporary male workers worked an average of
40 hours/a week $(\mathrm{p}<0.001$; data not shown in table). Since the temporary workers included daily workers or part-time workers, it would make sense that the routine activities and the standardized work patterns of permanent workers affect their outdoor activities and exposure to sunlight, rather than interpreting the permanent workers as the risk group for vitamin D deficiency.

Meanwhile, in our study, we failed to show a correlation between working hours and vitamin D deficiency. We believe that this result might have arisen from the fact that the subjects reported the number of their working hours as an answer to an open-ended question, which was subjective. Moreover, the exposure to sunlight, which is not necessarily work-related, might differ among individuals. Therefore, the number of working hours should also be investigated while taking into account other factors.

In the females, it was not possible to confirm a clear correlation between vitamin D levels and working conditions. The majority of the female workers $(84.7 \%)$ reported that they worked under the maximum number of legal working hours (52 hours/week), while the proportion of those working part-time jobs, which was $25.1 \%$, was also significantly higher than that of the male subjects. This signified that, among the female subjects, other variables beyond occupational conditions, such as quantity of housework, affect sunlight exposure and thus, in turn, vitamin D levels [32,33].

Opinions are conflicting regarding the appropriate cut-off values for defining the optimal levels of vitamin D. Men and women differ in terms of body size, muscle mass, and body fat, so their vitamin D metabolism and 
storage are also different [34-36]. The men in our study were more actively engaged in outdoor activities, while the women used cosmetics or sunscreen, which could have added another factor explaining the differences. Broader discussion is needed on whether it is appropriate to apply the same cut-off value of $20 \mathrm{ng} / \mathrm{mL}$ vitamin $\mathrm{D}$ to both genders. As women have tended to show a much higher prevalence of vitamin $\mathrm{D}$ deficiency when the cut-off value was set at $20 \mathrm{ng} / \mathrm{mL}$, it could be appropriate to apply a lower value for females.

Recently, many studies have supported the necessity of vitamin D management. Vitamin D plays a crucial role in preventing rickets or osteoporosis by helping to store calcium in the bones. One of the most serious forms of complications of vitamin D deficiency is osteomalacia, which is caused by low blood calcium and phosphate levels. Moreover, subclinical vitamin D deficiency could result in a non-symptomatic drop in bone density [37].

Previous reports on the correlation of vitamin D with cardiovascular disorders, cancers, and non-musculoskeletal disease, apart from its already-known effects on musculoskeletal systems, indicate the need for more proactive efforts to manage vitamin D deficiency [19,23,38-40]. Vitamin D has, moreover, been demonstrated to affect certain genes regulating blood pressure, increase the calcium levels in human cells, and stimulate immune cells to prevent inflammation or thrombosis in blood vessels [41,42]. Epidemiologic studies have reported that a person with vitamin D levels $\leq 15 \mathrm{ng} / \mathrm{mL}$ is at twice the risk of myocardial infarction over those with vitamin D levels $\geq 30 \mathrm{ng} / \mathrm{mL}$ [40]. Moreover, it has also been reported that people with vitamin $D$ levels of $\leq 15 \mathrm{ng} / \mathrm{mL}$ have a $2.7-8.1$ times higher risk of hypertension in the following 4-8 years [43]. There have been other reports suggesting that vitamin D deficiency is related to various cancers such as stomach, colon, breast, and prostate cancer, and it is believed that active vitamin D from various local tissues is capable of inducing cellular differentiation and the death of cancer cells $[44,45]$.

While few studies have been conducted on the consequences of vitamin D deficiency in wage workers specifically, recent reports have confirmed the correlation between vitamin $\mathrm{D}$ deficiency in workers and their Framingham score, a cardiovascular risk score [46]. In a cross-sectional study of 10,646 health care workers, a correlation between vitamin $\mathrm{D}$ levels and presenteeism (the problem of workers' being at their workplace but not fully functioning due to temporary illness or massive stress) was also confirmed, suggesting that vitamin D deficiency has the potential to undermine the productivity of workers [47].

It is generally sufficient to expose a portion of the body to sunlight for approximately $20 \mathrm{~min}$ a day to maintain sufficient levels of vitamin D $[38,48]$. However, if a worker works in shifts or is in the office the whole day, the opportunity for sunlight exposure will inevitably be decreased. In addition, numerous factors affect vitamin $\mathrm{D}$ production, including the weather, time of day, latitude, air pollution, sunblock use, and clothing. Therefore, simply recommending outdoor activities is not a satisfactory solution. In the absence of other options, dietary supplements fortified with vitamin D could be used as an alternative solution.

Foods with a high vitamin D level include fish such as salmon, anchovies, and mackerel, as well as dairy products such as milk. However, the maximum amount of vitamin $\mathrm{D}$ available in food is only approximately 100 IU/day [49]. The rest of the vitamin D requirement should be obtained from vitamin D supplements or milk products with fortified with additional vitamin D. Consumption of foods fortified with vitamin D may increase the daily intake by up to 800-1000 IU [37]. Therefore, dietary supplementation should be planned based on these facts in the workplace $[37,50]$.

This study has a few limitations. First, since this was a cross-sectional study, it was unclear whether the working conditions were responsible for the vitamin D deficiency. Second, the lack of information on exposure to sunlight (i.e., individual outdoor activity, sun exposure during work) and the amount of vitamin $\mathrm{D}$ consumed by the subjects limited the drawing of firm conclusions. Third, the Korea National Health and Nutrition Examination Survey was taken over 48 weeks a year at random times from January to December over 3 years, and the date of the examination was not disclosed for privacy. However, the amount of sunlight exposure is influenced by the seasons [51], and we could not control the data for seasonal factors, which may be important confounders.

While few studies have addressed the relationship between vitamin $\mathrm{D}$ deficiency and working conditions, this study was based on one of the most nationally representative data sets produced in Korea, from which it was possible to demonstrate the effects of working in shifts and office work on vitamin D deficiency. This study provided reasonable evidence of the need for vitamin $\mathrm{D}$ management in Korean wage workers. Since this study identified only the work-related factors that showed correlations with vitamin D deficiency, further studies are needed to supplement these findings, including studies focusing on complications of vitamin D deficiency (musculoskeletal/non-musculoskeletal) and actual effects on work productivity.

\section{Conclusion}

The increased prevalence of vitamin D deficiency is attracting more and more attention worldwide, and reports on its correlation with diseases other than musculoskeletal disorders, such as cardiovascular disorders and 
diabetes, have made it a popular issue in public health. While a number of reports have shown correlations between lack of sunlight exposure and vitamin D deficiency, few studies have examined the correlation between vitamin D deficiency and working conditions. Our analysis showed an association of shift work and office work with vitamin $\mathrm{D}$ deficiency. It should be noted, however, that the prevalence of vitamin D deficiency in all the subjects was very high. It is essential to manage the vitamin D status of wage workers to maintain the productivity of a company's entire workforce.

\section{Competing interests}

The authors declare that they have no competing interests.

\section{Authors' contributions}

HRJ designed the study and the analytic strategy. DSK, JTP and MYK supervised the research concept and design, acquisition of data. SJH, YJH and HSC analyzed the data and helped conduct the literature review. All authors read and approved the final manuscript.

\section{Acknowledgements}

There is no conflict of interest or financial support to declare.

\section{Author details}

'Department of Occupational Medicine, Korea University Hosipital, 123, Jeokgeum-ro, Ansan, Gyeonggido, Republic of Korea. ${ }^{2}$ Department of Occupational Medicine, Wonjin green hospital, 49-53, Sagajeong-ro, Jungnang-gu, Seoul, Republic of Korea.

Received: 7 March 2014 Accepted: 3 September 2014 Published online: 16 September 2014

\section{References}

1. Choi HS: Vitamin D Status in Korea. Endocrinol Metab (Seoul) 2013, 28:12-16.

2. Holick MF, Chen TC: Vitamin D deficiency: a worldwide problem with health consequences. Am J Clin Nutr 2008, 87:1080S-1086S.

3. van der Wielen RP, Lowik MR, van den Berg H, de Groot LC, Haller J, Moreiras $\mathrm{O}$, van Staveren WA: Serum vitamin D concentrations among elderly people in Europe. Lancet 1995, 346:207-210

4. Kulie T, Groff A, Redmer J, Hounshell J, Schrager S: Vitamin D: an evidencebased review. J Am Board Fam Med 2009, 22:698-706.

5. Hosseinpanah F, Pour SH, Heibatollahi M, Moghbel N, Asefzade S, Azizi F: The effects of air pollution on vitamin D status in healthy women: a cross sectional study. BMC Public Health 2010, 10:519.

6. Le Goaziou MF, Bodier E, Souweine G, Moreau A, Perdrix C, Flori M, Dupraz C: Musculoskeletal chronic pains and Vitamin D deficiency. Study before after in general practice. Presse Med 2013, 42:e106-e113.

7. Wepner F, Scheuer R, Schuetz-Wieser B, Machacek P, Pieler-Bruha E, Cross HS, Hahne J, Friedrich M: Effects of vitamin D on patients with fibromyalgia syndrome: a randomized placebo-controlled trial. Pain 2014, 155:261-268.

8. Ashtari F, Ajalli M, Shaygannejad V, Akbari M, Hovsepian S: The relation between Vitamin $D$ status with fatigue and depressive symptoms of multiple sclerosis. J Res Med Sci 2013, 18:193-197.

9. Antiel RM, Caudill JS, Burkhardt BE, Brands CK, Fischer PR: Iron insufficiency and hypovitaminosis $D$ in adolescents with chronic fatigue and orthostatic intolerance. South Med J 2011, 104:609-611.

10. Hoeck AD, Pall ML: Will vitamin D supplementation ameliorate diseases characterized by chronic inflammation and fatigue? Med Hypotheses 2011, 76:208-213.

11. Wang JHJ-CK: The association between shift work and bone mineral density: analysis of 2008-2009 Korean National Health and Nutrition Examination Survey. Korean J Occup Environ Med 2012, 24:274-286.

12. Sarikaya S, Ozdolap S, Mungan AG, Gumustas S, Koc U, Guven B, Begendik $F$ : Effect of underground working on vitamin $D$ levels and bone mineral densities in coal miners: a controlled study. J Int Med Res 2006, 34:362-366.
13. Puligheddu M, Conti S, Campagna M, Meloni M, Pau M, Cocco P, Marrosu F: [Cancer risk among shift workers: a review]. G Ital Med Lav Ergon 2012. 34:624-626

14. Ward M, Berry DJ, Power C, Hypponen E: Working patterns and vitamin D status in mid-life: a cross-sectional study of the 1958 British birth cohort. Occup Environ Med 2011, 68:902-907.

15. Itoh H, Weng Z, Saito H, Ogawa Y, Nakayama K, Hasegawa-Ohira M, Morimoto K, Maki S, Takahashi M: Association between night-shift work and serum 25-hydroxyvitamin D levels in Japanese male indoor workers: a cross-sectional study. Ind Health 2011, 49:658-662.

16. LeBoff MS, Kohlmeier L, Hurwitz S, Franklin J, Wright J, Glowacki J: Occult vitamin $D$ deficiency in postmenopausal US women with acute hip fracture. JAMA 1999, 281:1505-1511.

17. Lips P, Graafmans WC, Ooms ME, Bezemer PD, Bouter LM: Vitamin D supplementation and fracture incidence in elderly persons. A randomized, placebo-controlled clinical trial. Ann Intern Med 1996, 124:400-406.

18. Jackson RD, LaCroix AZ, Gass M, Wallace RB, Robbins J, Lewis CE, Bassford T, Beresford SA, Black HR, Blanchette P, Bonds DE, Brunner RL, Brzyski RG, Caan B, Cauley JA, Chlebowski RT, Cummings SR, Granek I, Hays J, Heiss G, Hendrix SL, Howard BV, Hsia J, Hubbell FA, Johnson KC, Judd H, Kotchen JM, Kuller LH, Langer RD, Lasser NL, et al: Calcium plus vitamin $\mathrm{D}$ supplementation and the risk of fractures. N Engl J Med 2006, 354:669-683.

19. Lee JH, O'Keefe JH, Bell D, Hensrud DD, Holick MF: Vitamin D deficiency an important, common, and easily treatable cardiovascular risk factor? J Am Coll Cardiol 2008, 52:1949-1956.

20. Wacker M, Holick MF: Sunlight and Vitamin D: A global perspective for health. Dermato-endocrinology 2013, 5:51-108.

21. Morris HA: Vitamin D: a hormone for all seasons-how much is enough? Clin Biochem Rev / Aust Assoc Clin Biochem 2005, 26:21-32.

22. Hossein-nezhad A, Holick MF: Vitamin D for health: a global perspective. Mayo Clin Proc 2013, 88:720-755.

23. Wang TJ, Pencina MJ, Booth SL, Jacques PF, Ingelsson E, Lanier K, Benjamin EJ, D'Agostino RB, Wolf M, Vasan RS: Vitamin D deficiency and risk of cardiovascular disease. Circulation 2008, 117:503-511.

24. Souberbielle JC, Body JJ, Lappe JM, Plebani M, Shoenfeld Y, Wang TJ, Bischoff-Ferrari HA, Cavalier E, Ebeling PR, Fardellone P, Gandini S, Gruson D, Guérin AP, Heickendorff L, Hollis BW, Ish-Shalom S, Jean G, von Landenberg P, Largura A, Olsson T, Pierrot-Deseilligny C, Pilz S, Tincani A, Valcour A, Zittermann A: Vitamin D and musculoskeletal health, cardiovascular disease, autoimmunity and cancer: Recommendations for clinical practice. Autoimmun Rev 2010, 9:709-715.

25. Hollis BW, Wagner CL: Normal serum vitamin D levels. N Engl J Med 2005, 352:515-516. author reply 515-516.

26. Holick MF: High prevalence of vitamin D inadequacy and implications for health. Mayo Clin Proc 2006, 81:353-373.

27. Malabanan A, Veronikis IE, Holick MF: Redefining vitamin D insufficiency. Lancet 1998, 351:805-806.

28. Thomas MK, Lloyd-Jones DM, Thadhani RI, Shaw AC, Deraska DJ, Kitch BT, Vamvakas EC, Dick IM, Prince RL, Finkelstein JS: Hypovitaminosis D in medical inpatients. N Engl J Med 1998, 338:777-783.

29. DeLuca HF: Overview of general physiologic features and functions of vitamin D. Am J Clin Nutr 2004, 80:1689S-1696S.

30. Haddad JG, Matsuoka LY, Hollis BW, Hu YZ, Wortsman J: Human plasma transport of vitamin D after its endogenous synthesis. J Clin Invest 1993, 91:2552-2555.

31. Sayre RM, Dowdy JC: Darkness at noon: sunscreens and vitamin D3. Photochem Photobiol 2007, 83:459-463.

32. Lopes JB, Danilevicius CF, Takayama L, Caparbo VF, Scazufca M, Bonfa E, Pereira RM: Vitamin D insufficiency: a risk factor to vertebral fractures in community-dwelling elderly women. Maturitas 2009, 64:218-222.

33. Nakamura K, Tsugawa N, Saito T, Ishikawa M, Tsuchiya Y, Hyodo K, Maruyama K, Oshiki R, Kobayashi R, Nashimoto M, Yoshihara A, Ozaki R, Okano T, Yamamoto M: Vitamin D status, bone mass, and bone metabolism in home-dwelling postmenopausal Japanese women: Yokogoshi Study. Bone 2008, 42:271-277.

34. Johnson LK, Hofso D, Aasheim ET, Tanbo T, Holven KB, Andersen LF, Roislien $\mathrm{J}$, Hjelmesaeth J: Impact of gender on vitamin D deficiency in morbidly obese patients: a cross-sectional study. Eur J Clin Nutr 2012, 66:83-90.

35. Lim JS, Kim KM, Rhee Y, Lim SK: Gender-dependent skeletal effects of vitamin D deficiency in a younger generation. J Clin Endocrinol Metab 2012, 97:1995-2004. 
36. Lagunova Z, Porojnicu AC, Lindberg F, Hexeberg S, Moan J: The dependency of vitamin $D$ status on body mass index, gender, age and season. Anticancer Res 2009, 29:3713-3720.

37. Holick MF: Vitamin D deficiency. N Engl J Med 2007, 357:266-281.

38. Holick MF: Vitamin D: importance in the prevention of cancers, type 1 diabetes, heart disease, and osteoporosis. Am I Clin Nutr 2004, 79:362-371.

39. Zittermann A: Vitamin D and disease prevention with special reference to cardiovascular disease. Prog Biophys Mol Biol 2006, 92:39-48.

40. Giovannucci E, Liu Y, Hollis BW, Rimm EB: 25-hydroxyvitamin D and risk of myocardial infarction in men: a prospective study. Arch Intern Med 2008, 168:1174-1180.

41. Lind L, Hanni A, Lithell H, Hvarfner A, Sorensen OH, Ljunghall S: Vitamin D is related to blood pressure and other cardiovascular risk factors in middle-aged men. Am J Hypertens 1995, 8:894-901.

42. Kunutsor SK, Burgess S, Munroe PB, Khan H: Vitamin D and high blood pressure: causal association or epiphenomenon? Eur J Epidemiol 2014, 29:1-14.

43. Forman JP, Giovannucci E, Holmes MD, Bischoff-Ferrari HA, Tworoger SS, Willett WC, Curhan GC: Plasma 25-hydroxyvitamin D levels and risk of incident hypertension. Hypertension 2007, 49:1063-1069.

44. Garland CF, Garland FC, Gorham ED, Lipkin M, Newmark H, Mohr SB, Holick MF: The role of vitamin D in cancer prevention. Am J Public Health 2006, 96:252-261.

45. Holick MF: Vitamin D: its role in cancer prevention and treatment. Prog Biophys Mol Biol 2006, 92:49-59.

46. Seung-Hyun Park J-HK: The relationship of serum vitamin D levels and the Framingham risk score among male workeres in the manufacturing sector. Korean J Occup Environ Med 2012, 24:115-123.

47. Plotnikoff GA, Finch MD, Dusek JA: Impact of vitamin D deficiency on the productivity of a health care workforce. J Occup Environ Med/Am College Occup Environ Med 2012, 54:117-121.

48. Hollis BW: Circulating 25-hydroxyvitamin D levels indicative of vitamin D sufficiency: implications for establishing a new effective dietary intake recommendation for vitamin D. J Nutr 2005, 135:317-322.

49. Bertone-Johnson ER, Powers SI, Spangler L, Brunner RL, Michael YL, Larson JC, Millen AE, Bueche MN, Salmoirago-Blotcher E, Liu S, Ockene JK, Ockene I, Manson JE: Vitamin D intake from foods and supplements and depressive symptoms in a diverse population of older women. Am J Clin Nutr 2011, 94:1104-1112

50. Holick MF, Binkley NC, Bischoff-Ferrari HA, Gordon CM, Hanley DA, Heaney RP, Murad MH, Weaver CM, Endocrine S: Evaluation, treatment, and prevention of vitamin D deficiency: an Endocrine Society clinical practice guideline. J Clin Endocrinol Metab 2011, 96:1911-1930.

51. Rosecrans $R$, Dohnal JC: Seasonal vitamin $D$ changes and the impact on health risk assessment. Clin Biochem 2014, 47(7-8):670-672

doi:10.1186/s40557-014-0028-x

Cite this article as: Jeong et al:: Vitamin D status and associated occupational factors in Korean wage workers: data from the 5th Korea national health and nutrition examination survey (KNHANES 2010-2012).

Annals of Occupational and Environmental Medicine 2014 26:28.

\section{Submit your next manuscript to BioMed Central and take full advantage of:}

- Convenient online submission

- Thorough peer review

- No space constraints or color figure charges

- Immediate publication on acceptance

- Inclusion in PubMed, CAS, Scopus and Google Scholar

- Research which is freely available for redistribution

Submit your manuscript at www.biomedcentral.com/submit
C Biomed Central 